\title{
Pulmonary manifestations in adult patients with chronic granulomatous disease
}

\author{
Hélène Salvator ${ }^{1,2,3}$, Nizar Mahlaoui ${ }^{4,5,6,7}$, Emilie Catherinot ${ }^{1}$, Elisabeth Rivaud ${ }^{1}$ \\ Benoit Pilmis $^{8}$, Raphael Borie ${ }^{9,10}$, Bruno Crestani ${ }^{9,10}$, Colas Tcherakian ${ }^{1,2,3}$, \\ Felipe Suarez ${ }^{4,6,7,11}$, Bertrand Dunogue ${ }^{12}$, Marie-Anne Gougerot-Pocidalo ${ }^{13,14}$, \\ Margarita Hurtado-Nedelec ${ }^{13,14}$, Jean-François Dreyfus ${ }^{15}$, Isabelle Durieu ${ }^{16}$, \\ Fanny Fouyssac ${ }^{17}$, Olivier Hermine ${ }^{4,6,7,11}$, Olivier Lortholary 4,6,7,8, \\ Alain Fischer ${ }^{4,5,6,7,18}$ and Louis-Jean Couderc ${ }^{1,2,3}$
}

\begin{abstract}
Affiliations: 'Service de Pneumologie, Hôpital Foch, Suresnes, France. ${ }^{2}$ UPRES EA 220, Suresnes, France. ${ }^{3}$ Faculté des Sciences de la Santé Simone Veil, Université Versailles-Saint-Quentin-en-Yvelines, Versailles, France. ${ }^{4}$ CEREDIH, Centre de Référence des Déficits Immunitaires Héréditaires, Hôpital Universitaire NeckerEnfants Malades, Assistance Publique-Hôpitaux de Paris, Paris, France. ${ }^{5}$ Service d'Immunologie-Hématologie et Rhumatologie Pédiatrique, Hôpital Universitaire Necker-Enfants Malades, Assistance Publique-Hôpitaux de Paris, Paris, France. 'Université Paris Descartes-Sorbonne Paris Cité, Institut Imagine, Paris, France. ${ }^{7}$ INSERM UMR 1163, Paris, France. ${ }^{8}$ Service des Maladies Infectieuses et Tropicales, Centre d'Infectiologie Necker Pasteur, Hôpital Necker-Enfants Malades, Assistance Publique-Hôpitaux de Paris, Paris, France. ${ }^{9}$ Service de Pneumologie A, Centre de Compétence Maladies Rares Pulmonaires, DHU FIRE, Assistance Publique-Hôpitaux de Paris, Hôpital Bichat, Paris, France. ${ }^{10}$ INSERM UMR 1152, Paris, France. ${ }^{11}$ Service d'Hématologie Adulte, Hôpital Necker-Enfants Malades, Assistance Publique-Hôpitaux de Paris, Paris, France. ${ }^{12}$ Service de Médecine Interne, Hôpital Cochin, Assistance Publique-Hôpitaux de Paris, Paris, France. ${ }^{13}$ UF Dysfonctionnements Immunitaires, Hôpital Bichat, Assistance Publique-Hôpitaux de Paris, Paris, France. ${ }^{14}$ INSERM UMR 1149, Paris, France. ${ }^{15}$ Délégation pour la Recherche Clinique et l'Innovation, Hôpital Foch, Suresnes, France. ${ }^{16}$ Service de Médecine Interne, Hospices Civils de Lyon, Groupe Hospitalier Sud, Université de Lyon, Lyon, France. ${ }^{17}$ Service d'Hématologie et Oncologie pédiatrique, Centre Hospitalier Universitaire de Nancy, Nancy, France. ${ }^{18}$ Collège de France, Paris, France.
\end{abstract}

Correspondence: Hélène Salvator, Service de Pneumologie, Hôpital Foch, 40 rue Worth, 92150 Suresnes, France. E-mail: h.salvatorahopital-foch.com

ABSTRACT Chronic granulomatous disease (CGD) is a primary immunodeficiency caused by failure of superoxide production in phagocytic cells. The disease is characterised by recurrent infections and inflammatory events, frequently affecting the lungs. Improvement of life expectancy now allows most patients to reach adulthood. We aimed to describe the pattern of pulmonary manifestations occurring during adulthood in CGD patients.

This was a retrospective study of the French national cohort of adult patients ( $\geqslant 16$ years old) with CGD.

Medical data were obtained for 67 adult patients. Pulmonary manifestations affected two-thirds of adult patients. Their incidence was significantly higher than in childhood (mean annual rate 0.22 versus 0.07 , $\mathrm{p}=0.01$ ). Infectious risk persisted despite anti-infectious prophylaxis. Invasive fungal infections were frequent ( 0.11 per year per patient) and asymptomatic in $37 \%$ of the cases. They often required lung biopsy for diagnosis (10 out of 30). Noninfectious respiratory events concerned $28 \%$ of adult patients, frequently associated with a concomitant fungal infection (40\%). They were more frequent in patients with the X-linked form of CGD. Immune-modulator therapies were required in most cases (70\%).

Respiratory manifestations are major complications of CGD in adulthood. Noninfectious pulmonary manifestations are as deleterious as infectious pneumonia. A specific respiratory monitoring is necessary.

@ERSpublications

Pulmonary involvement is a major concern in adult CGD patients, making specific respiratory monitoring necessary http://ow.ly/FjaRS

Editorial comment in Eur Respir J 2015; 45: 1521-1523 [DOI: 10.1183/09031936.00020215].

Received: June 272014 | Accepted after revision: Nov 262014 | First published online: Jan 222015

Support statement: This work was supported by LVL Medical (a grant of $€ 10000$ to H. Salvator). The funder had no role in study design, data collection and analysis, decision to publish, or preparation of the manuscript.

Conflict of interest: Disclosures can be found alongside the online version of this article at erj.ersjournals.com

Copyright @ERS 2015 


\section{Introduction}

Chronic granulomatous disease (CGD) is a rare inherited primary immunodeficiency caused by a genetic defect of the nicotinamide adenine dinucleotide phosphate (NADPH) oxidase. The mode of inheritance can be X-linked (mutations in the $C Y B B$ gene coding for gp $91^{\text {phox }}$ protein) or of autosomal recessive form (mutations in the NCF1, NCF2, CYBA or NCF4 genes coding for $\mathrm{p} 47^{\text {phox }}, \mathrm{p} 67^{\text {phox }}, \mathrm{p} 22^{\text {phox }}$ or $\mathrm{p} 40^{\text {phox }}$, respectively) [1]. This defect renders phagocytic cells unable to produce reactive oxygen species (ROS), which are crucial to killing ingested microorganisms. Thus, CGD is characterised by recurrent life-threatening infections as well as inflammatory manifestations $[2,3]$.

The diagnosis of CGD is usually evoked at an early age because of repeated infections [4]. The rate of severe infections is estimated to be 0.3 per patient per year $[1,4,5]$. CGD patients are particularly susceptible to fungal infections [6,7]. In a previous report on the French cohort of 155 CGD patients, $75 \%$ of them had presented at least one invasive fungal infection by the age of 30 years [8]. Chronic noninfectious events may occur in various organs $[9,10]$ : Crohn's-like colitis, digestive or urinary tract obstruction, ocular involvement or respiratory disorders $[9,11]$.

The lung is the most frequently affected organ (40-85\% of patients, both children and adults) [4, 5, 12-14]. Pulmonary infections are of severe concern and invasive aspergillosis represents the major cause of mortality [15]. Respiratory noninfectious manifestations are probably underestimated because of diagnostic difficulties. The presence of granuloma was reported in all 32 surgical lung resection tissues from CGD patients [16].

The outcome of CGD patients has dramatically improved over the last decades thanks to anti-infectious prophylaxis with itraconazole and trimethoprim-sulfamethoxazole [17, 18]. Patients now easily reach adulthood $[19,20]$ and CGD is no longer only a paediatric disease. However, little is known about the clinical presentation of respiratory events in adulthood. Therefore, the aim of our study was to identify the pattern of pulmonary involvement in adult CGD patients.

\section{Methods}

Patients

We conducted a retrospective study of the cohort of CGD adult patients included in the registry of CEREDIH, the French national reference centre for primary immunodeficiencies, which coordinates a national network of 60 medical departments [21]. After informed consent was obtained, the medical data of these patients was recorded in the French and European registries [22].

As of July 2011, 99 out of 174 registered patients with CGD had reached adulthood ( $\geqslant 16$ years old). Medical data were obtained for 74 of them. Seven patients were omitted because of incomplete data or being lost to follow-up for $>5$ years, resulting in 67 included patients.

Diagnosis of CGD was made using functional tests evaluating the production of ROS by neutrophils, mainly the nitro blue tetrazolium (NBT) test, and confirmed by genetic analysis (49 out of 67 available).

\section{Pulmonary analysis}

A specific questionnaire was created, recording: 1) general information on CGD history (age at diagnosis, functional and genetic results, allogeneic haematopoietic stem cell transplantation, and date of last follow-up); 2) number of respiratory infections and noninfectious events in childhood; 3) medical reports of all respiratory infections and noninfectious events in adulthood (age at the event, anti-infectious prophylaxis, clinical signs, biological results at diagnosis, thoracic computed tomography (CT) performed at diagnosis and after ending treatment, microbiological identification, diagnostic procedures, pulmonary functional tests, analysis of bronchoalveolar lavage fluid, and anti-infectious regimen and associated treatment (immunomodulator therapies and surgery)).

Invasive pulmonary fungal infections were classified according to the 2008 European Organization for Research and Treatment of Cancer and Mycoses Study Group criteria [23]. Noninfectious respiratory events were defined as any new pulmonary radiological opacity with: 1) spontaneous resolution without any anti-infectious treatment; 2) inflammatory pattern in a histological sample in the absence of detection of any pathogen; or 3) inflammatory pattern in a histological sample with an identified pathogen but unsatisfactory evolution with anti-infectious treatment only. Special care was taken in the search for pathogens in pulmonary and blood samples using all available techniques at the time of diagnosis.

\section{Statistical analysis}

Data are presented as mean \pm SD or median (range) as appropriate. Nonparametric comparative tests were performed using NCSS 9.0.7 (NCSS LLC, Kaysville, UT, USA) and GraphPad Prism version 5.0 (GraphPad Software, San Diego, CA, USA). Statistical significance was defined as p-values $<0.05$. 
Distinctive times of follow-up were taken into account using Poisson's regression. A subset selection algorithm allowed selection of the best parsimonious model, i.e. a model that only included regression coefficients with significant Wald probabilities for independent variables (including age at diagnosis, inheritance, NBT test results, number of infectious and noninfectious pulmonary events before and after the age of 16 years). When no such model existed a hierarchical forward selection model was used.

\section{Results}

\section{Characteristics of the patients}

We collected medical data for 67 adult patients with CGD: 57 males and 10 females (table 1). Mode of inheritance was X-linked for $47(70 \%)$ patients, autosomal recessive for 15 (22\%) patients and unknown for five (8\%) patients. No female carriers were included. NBT test results were available for 57 patients, and were null for $43(75 \%)$ patients, $>10 \%$ for five (9\%) patients and intermediate in nine (16\%) patients. One of the females presented an X-linked form of CGD due to an extreme and skewed X-inactivation (NBT test result 37\%).

Median age at diagnosis of CGD was 3.6 years (range 0-39 years). Four patients were diagnosed later than 16 years of age: two autosomal recessive and two X-linked (age at diagnosis 21, 22, 30 and 39 years; NBT test result 4\%,37\%, $0 \%$ and unknown, respectively). Median age at last follow-up was 25 years (range 1759 years). Median duration of follow-up in adulthood was 9 years (range 1-43 years).

Nine patients died (median age 24 years, range 17-46 years; X-linked form, eight out of nine patients). The cause of death was cerebral fungal abscess in three cases (due to Aspergillus nidulans, Rasamsonia argillacea and one unidentified fungal agent), respiratory infections in three cases (due to Streptococcus constellatus, Klebsiella pneumoniae and Aspergillus fumigatus), Burkholderia cepacia septicaemia in one case and chronic respiratory failure. The cause of death was unknown in one case.

Four patients underwent stem-cell transplantation in childhood for refractory respiratory infectious or inflammatory manifestations. They were cured after transplantation and did not present any pulmonary complication during adulthood. Their evolution is consistent with published data that mention good outcome after haematopoietic stem-cell transplantation for CGD [24, 25]. We excluded them from statistical analyses so as not to underevaluate pulmonary manifestations.

\section{Incidence and risk factors of pulmonary manifestations in adulthood}

Most patients $(52(82 \%)$ out of 63 ) presented at least one respiratory manifestation during the whole assessed period. During adulthood specifically, two-thirds of the patients (42 (67\%) out of 63) presented one or more respiratory event.

Respiratory complications were more frequent in adulthood than in childhood (mean $\pm \mathrm{SD}$ annual rate $0.22 \pm 0.37$ versus $0.07 \pm 0.08, \mathrm{p}=0.01$ ), especially fungal infections and noninfectious events but not bacterial infections (table 2).

\begin{tabular}{|c|c|c|c|c|}
\hline & Total & X-linked & AR & UNK \\
\hline Patients & 67 & $47(70)$ & $15(22)$ & $5(8)$ \\
\hline \multicolumn{5}{|l|}{ Genetic pattern } \\
\hline CYBB (gp $91^{\text {phox }}$ ) & & 47 & & \\
\hline NCF1 (p47 ${ }^{\text {phox }}$ ) & & & 10 & \\
\hline$C Y B A\left(\mathrm{p} 22^{\text {phox }}\right]$ & & & 3 & \\
\hline NCF2 (p67 $\left.7^{\text {phox }}\right)$ & & & 2 & \\
\hline Males/females & $57 / 10$ & $46 / 1$ & $7 / 8$ & $4 / 1$ \\
\hline Deaths & 9 & 8 & 0 & 1 \\
\hline Age at diagnosis years & $3.6(0-39)$ & $3.2(0-39)$ & $4.3(0-30)$ & $1.5(0.75-12)$ \\
\hline Age at last follow-up years & $25(17-59)$ & $25(17-46)$ & $28.5(18-59)$ & $23(19-39)$ \\
\hline Duration of follow-up in adulthood years & $9(1-43)$ & $9(1-30)$ & $12(2-43)$ & $7(3-23)$ \\
\hline NBT test & 57 & 41 & 14 & 2 \\
\hline $0 \%$ & $43(75)$ & 29 & 13 & 1 \\
\hline $1-9 \%$ & $9(16)$ & 7 & 1 & 1 \\
\hline$\geqslant 10 \%$ & $5(9)$ & 5 & 0 & 0 \\
\hline
\end{tabular}

Data are presented as $\mathrm{n}(\%), \mathrm{n}$ or median (range), unless otherwise stated. AR: autosomal recessive; UNK: unknown mode of transmission. 
TABLE 2 Annual rate of respiratory events before and after the age of 16 years, considering inheritance pattern

\begin{tabular}{|c|c|c|c|c|c|c|}
\hline & \multicolumn{3}{|c|}{ Before the age of 16 years } & \multicolumn{3}{|c|}{ After the age of 16 years } \\
\hline & Total & $\mathbf{X L}$ & AR & Total & $\mathbf{X L}$ & AR \\
\hline Total respiratory infections & $0.06 \pm 0.07 *$ & $0.06 \pm 0.07 *$ & $0.05 \pm 0.06$ & $0.18 \pm 0.34^{*}$ & $0.19 \pm 0.36 *$ & $0.06 \pm 0.08$ \\
\hline Respiratory bacterial infections & $0.03 \pm 0.04$ & $0.03 \pm 0.04$ & $0.03 \pm 0.04$ & $0.07 \pm 0.14$ & $0.05 \pm 0.11$ & $0.04 \pm 0.07$ \\
\hline Noninfectious respiratory events & $0.01 \pm 0.02 * *$ & $0.01 \pm 0.02 * *$ & 0.00 & $0.05 \pm 0.14^{* *}$ & $0.06 \pm 0.17^{* *}$ & $0.01 \pm 0.02$ \\
\hline
\end{tabular}

Data are presented as mean \pm SD. Statistical analysis was performed in each group comparing annual rates before and after the age of 16 years. $\mathrm{XL}$ : X-linked; AR: autosomal recessive. ${ }^{*}: \mathrm{p}<0.05{ }^{* *}: \mathrm{p}<0.01$.

Time to first episode in adulthood was not statistically different between the X-linked and autosomal recessive groups (fig. 1). No statistical difference was highlighted in terms of global annual rates of pulmonary events from one age group to another (fig. 2).

CGD inheritance did not influence the occurrence of respiratory infections but X-linked CGD patients were at higher risk of developing a pulmonary noninfectious complication in adulthood (relative risk 5.55, 95\% CI 1.43-20; $\mathrm{p}=0.01$ ). NBT test results did not emerged as a risk factor for infectious or noninfectious respiratory events. Having previously presented similar events in childhood favoured the recurrence of fungal infections and noninfectious pulmonary events during adulthood $(p=0.02$ and $p=0.008$, respectively). Finally, the occurrence of fungal infections and noninfectious respiratory events during adulthood were statistically associated in a multivariate analysis $(\mathrm{p}=0.002)$.

\section{Characteristics of respiratory infections}

We recorded as many cases of fungal $(n=30)$ as of bacterial pneumonia $(n=30)$. Simultaneously, 70 extrarespiratory infections were recorded, especially cutaneous infections $(n=25)$, digestive infections $(\mathrm{n}=17)$ and adenitis $(\mathrm{n}=14)$.

\section{Bacterial pneumonia}

Fever or respiratory functional signs (dyspnoea, cough or purulent sputum) were present in $89 \%$ of bacterial pneumonia (table 3).

The identified pathogen was available in 19 (63\%) cases: mycobacteria in five (two cases of Mycobacterium tuberculosis, one of Mycobacterium bovis, one of both Mycobacterium mucogenicum and M. bovis, and one of Mycobacterium gordonae), B. cepacia in three, Nocardia farcinica in one, and Nocardia sp. in one, K. pneumoniae in one, S. constellatus in one, both Streptococcus salivarius and Veillonella in one, Staphylococcus aureus in one, Serratia marcescens in one, Mycoplasma pneumoniae in one, Salmonella (non-typhi) in one, Haemophilus influenzae in one, and Branhamella catarrhalis in one.

a)
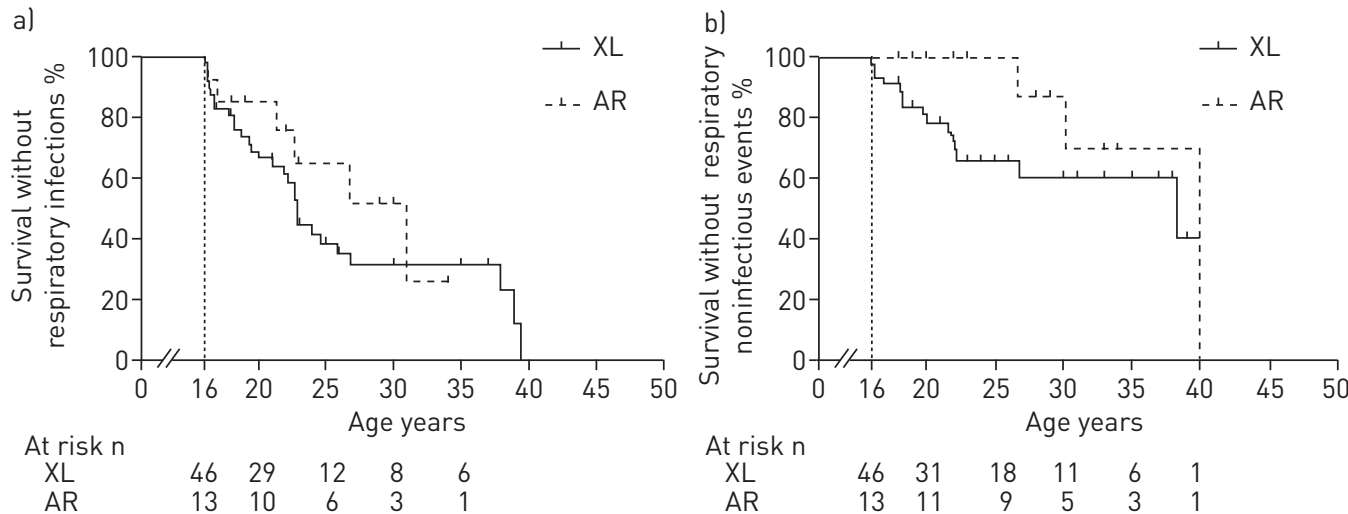

FIGURE 1 a) Survival without infectious respiratory complications from the age of 16 years (Kaplan-Meyer analysis) according to chronic granulomatous disease (CGD) inheritance (X-linked (XL) versus autosomal recessive (AR), $\mathrm{p}=0.3$ ). b) Survival without noninfectious respiratory complications from the age of 16 years (Kaplan-Meyer analysis) according to CGD inheritance (XL versus AR, $\mathrm{p}=0.07$ ). 

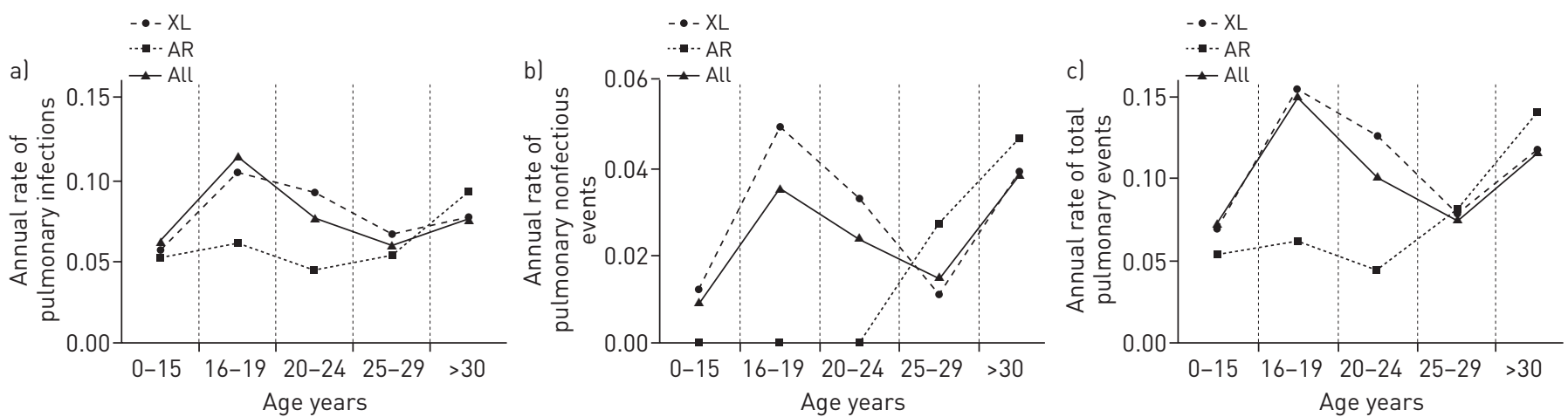

FIGURE 2 Annual rate of a) pulmonary infections, b) pulmonary noninfectious events and c) total pulmonary events as a function of age and inheritance mode of chronic granulomatous disease. Age: $0-15$ years, $n=63 ; 16-19$ years, $n=63 ; 20-24$ years, $n=51 ; 25-29$ years, $n=37 ;>30$ years, $n=22$. XL: X-linked; AR: autosomal recessive.

At diagnosis, thoracic CT showed alveolar consolidations (10 out of 13 patients), pulmonary nodules (six out of 13 patients), ground-glass opacities (five out of 13 patients), reticulations (two out of 13 patients), bronchiectasis (two out of 13 patients) and pleural effusion (two out of 13 patients).

TABLE 3 Infectious respiratory events: clinical, radiological and biological characteristics and treatments

Invasive fungal infection

\begin{tabular}{|c|c|c|}
\hline Events $\mathrm{n}$ & 30 & 30 \\
\hline Anti-infectious prophylaxis & Itraconazole & TMP-SMX \\
\hline Prescriptions & $24 / 29$ & $26 / 27$ \\
\hline \multicolumn{3}{|l|}{ Clinical signs at diagnosis $\mathrm{n} / \mathrm{N}(\%)$} \\
\hline Fever & $13 / 27(48)$ & $17 / 27(63)$ \\
\hline Respiratory function signs ${ }^{\#}$ & $13 / 27(48)$ & $17 / 27(63)$ \\
\hline Fever or respiratory function signs ${ }^{\#}$ & $17 / 27(63)$ & $24 / 27(89)$ \\
\hline Chest pain & $5 / 27(18.5)$ & $6 / 27(22)$ \\
\hline Respiratory failure & $2 / 27$ & $3 / 27$ \\
\hline \multicolumn{3}{|l|}{ Biology } \\
\hline PMNs $\times 10^{9}$ cells $\cdot L^{-1}$ & $6.0 \pm 3.5$ & $6.7 \pm 3$ \\
\hline $\mathrm{CRP} \mathrm{mg} \cdot \mathrm{L}^{-1}$ & $68 \pm 85$ & $106 \pm 90$ \\
\hline Platelets $\times 10^{9}$ cells $\cdot \mathrm{L}^{-1}$ & $297 \pm 117$ & $302 \pm 125$ \\
\hline Radiological features at diagnosis $n$ & 23 & 13 \\
\hline Nodule $\mathrm{n}$ & 12 & 6 \\
\hline Alveolar consolidation $\mathrm{n}$ & 20 & 10 \\
\hline Ground-glass opacities $n$ & 11 & 5 \\
\hline Reticulations $\mathrm{n}$ & 10 & 2 \\
\hline Bronchiectasis $n$ & 4 & 2 \\
\hline Radiological scarring after treatment & $9 / 9$ & $3 / 7$ \\
\hline Bronchoalveolar lavage $\mathrm{n}$ & 19 & 7 \\
\hline Cells $\times 10^{3}$ cells $\cdot \mathrm{mL}^{-1}$ & $675 \pm 1187$ & $419 \pm 372$ \\
\hline Macrophages \% & 43 & 38 \\
\hline Neutrophils \% & 23 & 21 \\
\hline Lymphocytes \% & 36 & 38 \\
\hline Eosinophils \% & 7 & 6 \\
\hline Pulmonary biopsy & $10 / 30$ & $4 / 30$ \\
\hline \multicolumn{3}{|l|}{ Treatment } \\
\hline Lines of treatment $\mathrm{n}$ mean & 2.2 & 1.7 \\
\hline Associated treatments $\mathrm{n}$ & 11 & 4 \\
\hline Interferon- $\gamma \mathrm{n}$ & 7 & 1 \\
\hline Granulocyte transfusion $n$ & 5 & 1 \\
\hline Surgery $n$ & 0 & 2 \\
\hline Immunomodulator $\mathrm{n}$ & 6 & 0 \\
\hline
\end{tabular}

Data are presented as $\mathrm{n} / \mathrm{N}$ or mean $\pm \mathrm{SD}$, unless otherwise stated. TMP-SMX: trimethoprim-sulfamethoxazole; PMN: polymorphonuclear neutrophil; CRP: C-reactive protein. ${ }^{\#}$ : dyspnoea, cough or purulent sputum. 
The therapeutic regimen consisted of drug combinations in a majority of cases, including mainly penicillin (24 patients), cephalosporin (11 patients), fluoroquinolone (10 patients), carbapenem (eight patients) and glycopeptide (eight patients). Antifungal treatments were added in eight cases for proven or suspected mixed infection. One patient received interferon- $\gamma$ and one patient had granulocyte transfusions. Surgical pleurocentesis was required in two patients.

Thoracic CT performed after the end of treatment $(n=7)$ and highlighted radiological sequelae in three cases. Two patients died from respiratory failure during the course of infection.

\section{Fungal infections}

$37 \%$ of the patients presented neither fever nor respiratory function signs (dyspnoea, cough or purulent sputum) at diagnosis of the fungal infection. These signs were each present in $48 \%$ of the cases. One patient presented haemoptysis (table 3 ).

The majority of patients (24 (83\%) out of 29) were receiving prophylaxis (itraconazole) at the time of the event. Serum itraconazole levels were below the recommended thresholds in five out of seven available cases.

Invasive fungal infections were classified as certain in seven out of 30 cases, probable in 11 out of 30 cases and possible in 12 out of 30 cases. Microbiological identification was obtained in 14 (46\%) cases: A. fumigatus in seven, A. nidulans in five, Aspergillus terreus in one and $R$. argillacea in one. At diagnosis, Aspergillus serological tests were positive in 22 (84\%) out of 26 cases while galactomannan antigenaemia was negative in all the 15 cases tested.

23 thoracic CTs were performed at diagnosis. They showed alveolar consolidations (20 out of 23 scans) (fig. 3), nodules (12 out of 23 scans), reticulations (10 out of 23 scans) and ground-glass opacities (11 out of 23 scans). Chest-wall invasion was detected in six cases: rib lysis (three cases), subcutaneous abscesses (two cases) and subclavian artery compression (one case).

Bronchoalveolar lavage fluid analysis was available in 19 cases, showing alveolitis (mean $675 \times 10^{3}$ cells. $\mathrm{mL}^{-1}$ ) with a mean of $43 \%$ macrophages, $36 \%$ lymphocytes, $23 \%$ neutrophils and $7 \%$ eosinophils. A fungal agent was identified in 10 (52\%) out of 19 of these samples. A pulmonary biopsy was performed in $10(33 \%)$ cases, disclosing fibrosis, epithelioid cells forming granuloma, and infiltration of lymphocytes, plasmocytes and neutrophils. A fungal agent was identified in six out of 10 cases.

Therapeutic management required a prolonged duration of treatment (median 307 days, range 30-730 days). Monotherapies were preferred (40 out of 68 lines of treatment), mainly intravenous amphotericin B deoxycholate before the year 2000 (seven out of seven) and voriconazole thereafter (14 out of 23). Drug-toxicity-related events led to a switch of drug in 10 cases: renal failure (three cases), cutaneous toxicity due to voriconazole (three cases), hepatitis (two cases), cytopenia (one case) and drug interaction (one case).

In addition to the antifungal therapies, seven patients received interferon- $\gamma$ and five granulocyte transfusions. Six patients received immune modulators, sometimes in association (corticosteroids (four patients), thalidomide (two patients) and hydroxychloroquine (two patients)).

Nine thoracic CTs were performed after the end of treatment, all showing radiological scarring (local fibrosis, septa thickenings and bronchiectasis) (fig. 4).
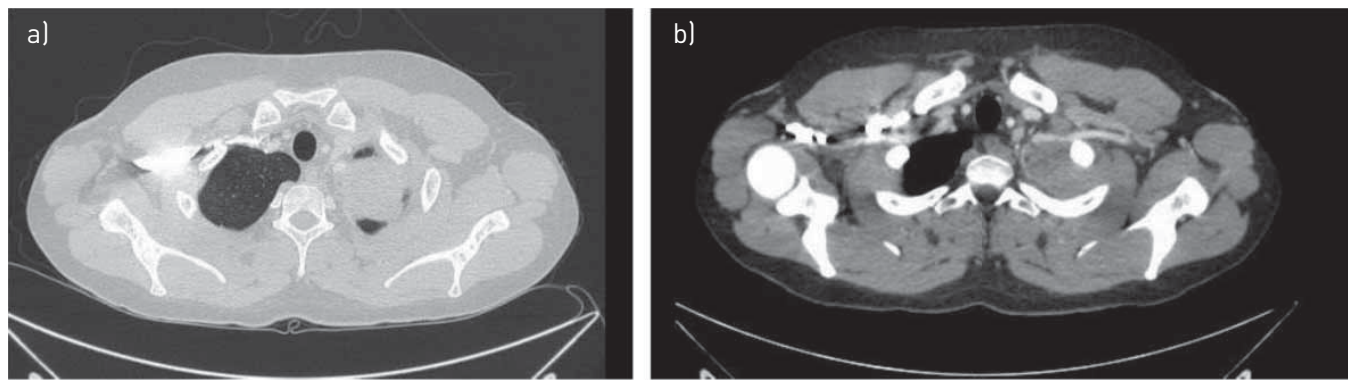

FIGURE 3 Pulmonary fungal invasive infection in a 25 -year-old man with chronic granulomatous disease. Contrast-enhanced computed tomography showed a left apical mass was responsible for subclavian artery compression. Pulmonary biopsy allowed Aspergillus nidulans identification. a) Left apical consolidation due to A. nidulans infection; b) compression of left subclavian artery by the fungal infectious mass. 

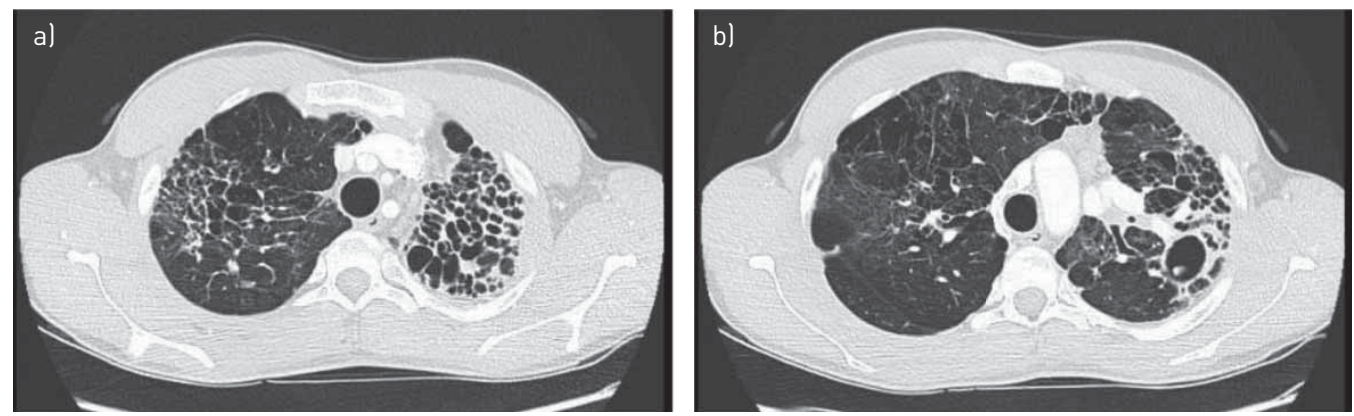

FIGURE 4 A 23-year-old man with chronic granulomatous disease presented cystic bronchiectasis (computed tomography images) due to repeated fungal infections in childhood. One of the cavities was infected by Aspergillus fumigatus. a) Cystic bronchiectasis in apical areas; b) infection by A. fumigatus of a residual cavity in the left upper lobe.

Four patients died because of invasive fungal infection: three from cerebral abscesses and one from respiratory failure.

Characteristics of noninfectious pulmonary events

20 noninfectious respiratory events occurred in 18 patients $>16$ years old. 13 (65\%) patients presented respiratory symptoms (dyspnoea or cough) at diagnosis (table 4).

In comparison, we recorded 60 extrathoracic noninfectious complications, principally digestive manifestations $(\mathrm{n}=26)$, cutaneous manifestations $(\mathrm{n}=16)$ or urinary tract involvement $(\mathrm{n}=5)$.

These events could be divided into two major types, depending on their radiological pattern, as follows. 1) Circumscribed nodules or parenchymal consolidation: seven of these 11 events occurred in the aftermath of a respiratory infection (six out of seven were aspergillosis). Pulmonary biopsy was frequently performed $(n=8)$, mainly showing granuloma (six out of eight) and neutrophilic or eosinophilic microabscesses (five out of eight). 2) Interstitial lung disease: only one of these nine events occurred in the aftermath of aspergillosis. The radiological pattern was variable: sarcoidosis-like in two cases (one case of disseminated micronodules and one case of peribronchial thickening in the upper lobes), nonspecific

TABLE 4 Noninfectious respiratory events: clinical, radiological, biological characteristics and treatments

\section{Total}

\begin{tabular}{cc}
\multicolumn{2}{c}{ Radiological pattern } \\
\hline $\begin{array}{c}\text { Interstitial lung } \\
\text { disease }\end{array}$ & $\begin{array}{c}\text { Nodules/ } \\
\text { parenchymal } \\
\text { consolidation }\end{array}$
\end{tabular}

\begin{tabular}{lccc}
\hline Events & 20 & 9 & 11 \\
Respiratory function signs & $13(65)$ & 6 & 7 \\
Concomitant infection & $8(40)$ & 1 & 7 \\
CT scan at diagnosis & & 3 & 7 \\
$\quad$ Consolidation & 10 & 1 & 5 \\
$\quad$ Nodule & 6 & 8 & 3 \\
$\quad$ Reticulations & 11 & 6 & 1 \\
$\quad$ Ground-glass opacities & 7 & 6 & 1 \\
$\quad$ Bronchiectasis & 7 & 2 & 8 \\
Biopsy & & 0 & 6 \\
$\quad$ Granuloma & & 0 & 5 \\
Eosinophil or neutrophil microabscesses & & 2 & 2 \\
$\quad$ Diffuse granulocyte infiltration without granuloma & 14 & & 4 \\
Anti-inflammatory therapies & 10 & 6 & 2 \\
$\quad$ Corticosteroids & 4 & 2 & 3 \\
$\quad$ Thalidomide & 4 & 1 & 0 \\
Hydroxychloroquine & 2 & 2 & \\
$\quad$ Methotrexate & & &
\end{tabular}

Data are presented as $\mathrm{n}$ or $\mathrm{n}(\%)$. CT: computed tomography. 
interstitial pneumonia in five cases (ground-glass opacities, reticulations and septa thickening), associated or not with scarring images (bronchiectasis, local retractile fibrosis and emphysema). Two of these patients were diagnosed with disproportionate pulmonary hypertension. Pulmonary biopsy was performed in two cases showing diffuse granulocyte infiltration, fibrosis and a discrete pattern of bronchiolitis but no granuloma. The presence of pigmented macrophages and a suggestive pattern of veno-occlusive disease were noted in one case [26].

Taking into account only events that were not associated with infection, bronchoalveolar lavage fluid analysis results were similar to those obtained during infectious manifestations (mean of $702 \times 10^{3}$ cells $\cdot \mathrm{mL}^{-1}$, 51\% macrophages, $28 \%$ lymphocytes, $20 \%$ neutrophils and $1 \%$ eosinophils).

Pulmonary function tests were available for 12 patients, demonstrating a restrictive pattern in seven cases, obstructive in two cases and normal values in three cases.

The management of these noninfectious respiratory events required immune modulator therapy in 14 (70\%) cases. The most frequently used drugs were: corticosteroids (10 cases), thalidomide (four cases) and hydroxychloroquine (four cases). Methotrexate was also prescribed in two cases and one patient benefited successively from intravenous immunoglobulins, mycophenolate mofetil, cyclophosphamide and rituximab. Overall, these treatments appeared effective on persistent consolidation associated with an infection but did not change the course of interstitial pneumonia. Persistent pulmonary involvement was noted on all the nine available thoracic CTs performed after the end of treatment.

\section{Discussion}

The aim of our study was to describe the pulmonary manifestations presented by adult CGD patients. To our knowledge, this is the largest series specifically focused on pulmonary complications in adults [27], based on the CEREDIH registry, the largest national registry for primary immune deficiencies worldwide. Our results are comparable to those of the main series of CGD patients regarding demographic data, transmission pattern, or rate of infections $[4,5]$.

First, we confirm that the lung is the main affected organ in CGD: $82 \%$ of patients presented at least one respiratory complication once in their life and $67 \%$ specifically during adulthood (versus $51 \%$ and $46 \%$ for digestive and cutaneous manifestations, respectively). An important point to outline is that there is no decrease in the frequency of respiratory events with age, meaning that physicians must remain particularly vigilant when monitoring these patients throughout their lives.

The majority of deaths (eight out of nine) occurred in the X-linked CGD patients, confirming the poor prognosis associated with this inherited trait [4]. The simple and rapid NBT test, performed in most of our patients, is not specific to superoxide anion production by NADPH oxidase. The use of a ferricytochrome $\mathrm{c}$ reduction assay could have been more helpful in highlighting differences between groups, as in the study by KuHNs et al. [28], where residual ROS production emerged as a predictor of survival in CGD patients.

Pulmonary fungal infections in CGD patients exhibit unusual features. Firstly, many of them were asymptomatic. This finding is consistent with a previous report from our group where only $55 \%$ of the 28 patients with fungal pneumonia had respiratory function signs and $38 \%$ had fever [29]. In the study by SEGAL et al. [30], one third of the patients with aspergillosis were asymptomatic. Secondly, we observed a high frequency of locoregional extension involving the chest wall (six cases), as previously reported [29, 30]. Finally, the fungal species involved are also infrequent. A. nidulans was involved in $16 \%$ of all invasive fungal infections in our series. This rate is lower than reported in the monocentric experience of BLUMENTAL et al. [29] (34.4\% of proven fungal infections), but consistent with the overall analysis of the French cohort (13.8\% of all fungal infections) [8]. A. nidulans is clearly over-represented in CGD compared with other immunodeficient states, suggesting differences in host-pathogen interactions and the role of NADPH oxidase pathway within Aspergillus species [31, 32].

A majority of infections occurred even though an anti-infectious prophylaxis was prescribed. The residual concentration of itraconazole was under recommended thresholds in most of the patients. This highlights the importance of regular monitoring of serum concentration of azole.

Little is known about the prevalence of noninfectious pulmonary manifestations in CGD patients. A retrospective study by our group [11], recording inflammatory manifestations in a single-centre cohort of 98 CGD patients (median age 13.5 years old), noted the occurrence of respiratory events in $26.4 \%$ of the patients (8.6\% of all inflammatory events recorded). LIESE et al. [20] reported, among a cohort of 39 patients (median age 12.9 years old), the presence of pulmonary fibrosis in $10 \%$ and pulmonary granuloma in $13 \%$ of the patients. In our series, noninfectious pulmonary events concerned $28 \%$ of the patients. They were more frequent in the X-linked inheritance group, even if the small size of the sample 
may overestimate their relative risk. $40 \%$ of these inflammatory events were concomitant with an infection, mainly aspergillosis. Pulmonary biopsy was then frequently required in order to differentiate them from uncontrolled infection, particularly in case of circumscribed nodules or parenchymal consolidation (nine out of 30).

In CGD, infection and chronic inflammation are clearly linked. Experimentally, fungal infections can activate uncontrolled pulmonary inflammation [33]. Recent studies have emphasised the role of ROS, such as anti-inflammatory mediators controlling the expression of various genes [34] and involved in the Treg/ Th17 lymphocyte balance [35].

Some infectious events may be confused with aseptic inflammatory flares. This diagnosis presents major therapeutic implications, such as immunomodulator prescription (mainly corticosteroids, thalidomide and hydroxychloroquine). Evaluation of the efficacy of these treatments is difficult to assess because of frequent co-prescription or association with antifungal therapies. We have previously reported our experience of thalidomide in inflammatory manifestations of CGD [36]. It was particularly effective on nodular and pseudo-mass lung lesions but ineffective for treating interstitial lung disease. As the infectious risk of steroids is a matter of concern in CGD patients, we think that thalidomide should be preferred as first-line treatment for noninfectious, evolutive pulmonary consolidation, but its use should be as short as possible in order to limit side-effects. Management of interstitial lung disease remains an unresolved issue. Efforts should be made to better characterise them. Perhaps fluorodeoxyglucose positron emission tomography could be helpful, as for pulmonary sarcoidosis, to distinguish active lesions from retractile interstitial sequelae [37].

Pulmonary complications are of poor prognosis (seven out of nine deaths related to respiratory complications) and frequently responsible for sequelae. Godoy et al. [38] found retractile scarring images in all the CTs of five adult CGD patients. The burden of respiratory complications in adulthood raises the question of specific respiratory monitoring of CGD patients. We propose a specific consultation once yearly,

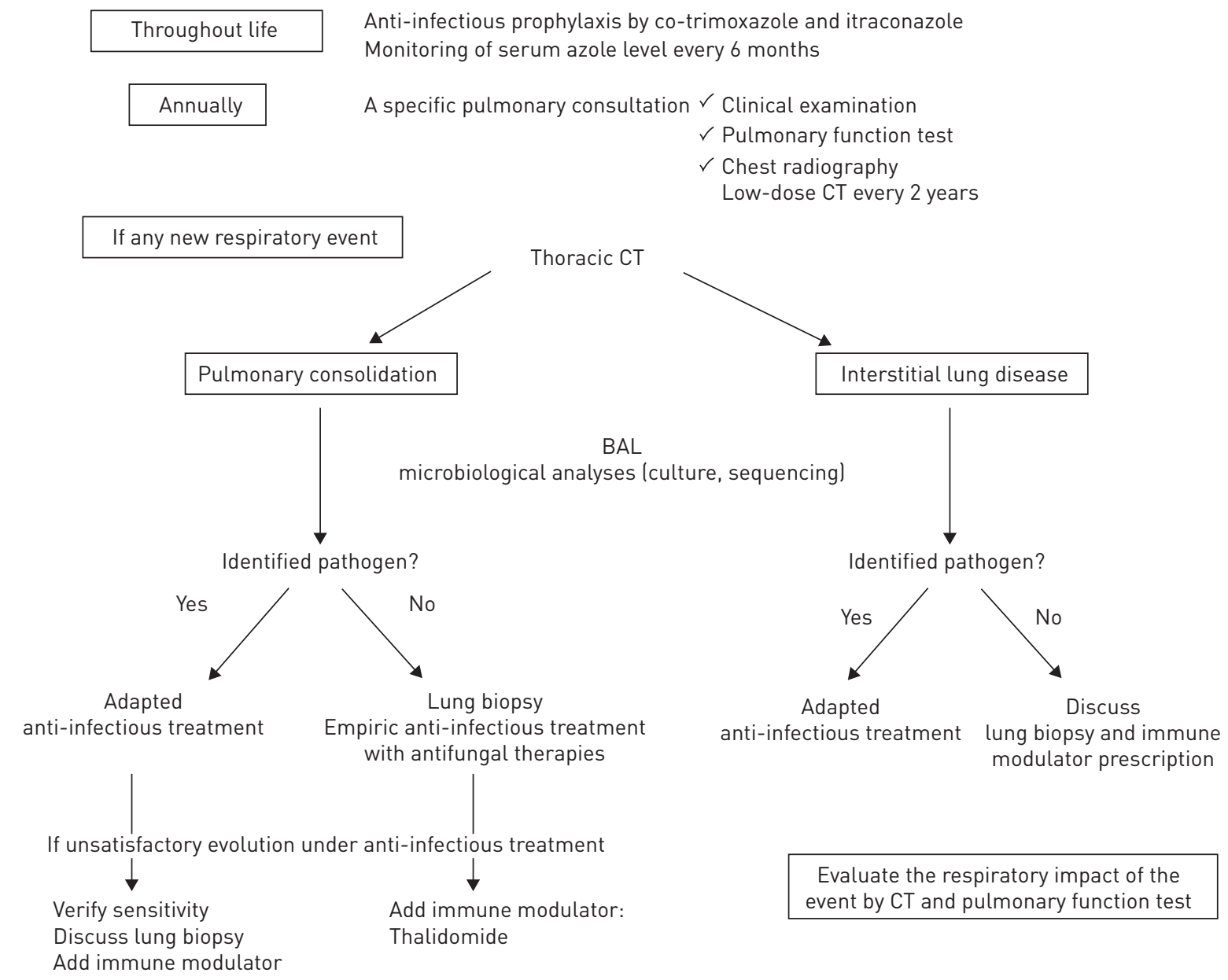

FIGURE 5 Guidelines for respiratory management of adult chronic granulomatous disease patients. BAL: bronchoalveolar lavage. 
with chest radiography and pulmonary functional tests. These are useful noninvasive means to monitor these patients and should be performed more frequently. A low-dose CT could be systematically performed every 2 years. In the case of any new respiratory event, management should be early and include invasive diagnostic techniques, even pulmonary biopsy. Anti-infectious drugs, particularly antifungals, are indicated as first-line treatment but immune modulator prescription should be considered early if the evolution remains unsatisfactory (fig. 5). Allogeneic bone-marrow transplantation may also be a therapeutic option in discussion with an immunologist in cases of severe and repeated respiratory complications. Restricted in the past to particularly severe cases in childhood, its indication is under discussion. Improvement in outcomes suggests that its indication should be extended and performed as early as possible in order to prevent the lifelong risk of complications [39, 40].

\section{Acknowledgements}

We thank Francois Mellot (Dept of Radiology, Foch Hospital, Suresnes, France) for reviewing thoracic CT images and Leila Zemoura (Dept of Pathology, Foch Hospital) for her help with the histological analysis of pulmonary biopsies. We thank Roland Jaussaud (Dept of Internal Medicine and Infectious Diseases, Robert Debré University Hospital, Reims, France), Jean-Charles Dalphin (Dept of Respiratory Diseases, Jean Minjoz University Hospital, Besançon, France), Gérard Michel (Dept of Pediatric Haematology, La Timone University Hospital, Marseille, France), Denis Vital-Durand (Dept of Internal Medicine, Lyon-Sud University Hospital, Lyon, France), Claudine Barbuat (Dept of Infectious Diseases, University Hospital, Nimes, France), Mathieu Revest (Dept of Infectious Diseases, University Hospital, Rennes, France) and Hervé Rubie (Dept of Pediatric Haematology, University Hospital, Toulouse, France) for providing access to medical data of CGD patients they have treated. Finally, we thank Cécile Mignot and Nathalie Devergnes (CEREDIH, University Necker-Enfants Malades Hospital, Paris, France) for their role in managing the CEREDIH registry.

\section{References}

$1 \quad$ Holland SM. Chronic granulomatous disease. Clin Rev Allergy Immunol 2010; 38: 3-10.

2 Segal BH, Leto TL, Gallin JI, et al. Genetic, biochemical, and clinical features of chronic granulomatous disease. Medicine 2000; 79: 170-200.

3 Stasia MJ, Cathebras P, Lutz M-F, et al. La granulomatose septique chronique [Chronic-granulomatous disease]. Rev Med Interne 2009; 30: 221-232.

4 Winkelstein JA, Marino MC, Johnston RB, et al. Chronic granulomatous disease. Report on a national registry of 368 patients. Medicine 2000; 79: 155-169.

5 Martire B, Rondelli R, Soresina A, et al. Clinical features, long-term follow-up and outcome of a large cohort of patients with chronic granulomatous disease: an Italian multicenter study. Clin Immunol 2008; 126: 155-164.

6 Henriet S, Verweij PE, Holland SM, et al. Invasive fungal infections in patients with chronic granulomatous disease. Adv Exp Med Biol 2013; 764: 27-55.

7 Falcone EL, Holland SM. Invasive fungal infection in chronic granulomatous disease: insights into pathogenesis and management. Curr Opin Infect Dis 2012; 25: 658-669.

8 Beauté J, Obenga G, Le Mignot L, et al. Epidemiology and outcome of invasive fungal diseases in patients with chronic granulomatous disease: a multicenter study in France. Pediatr Infect Dis J 2011; 30: 57-62.

9 Rosenzweig SD. Inflammatory manifestations in chronic granulomatous disease (CGD). J Clin Immunol 2008; 28: Suppl. 1, S67-S72.

10 Schäppi MG, Jaquet V, Belli DC, et al. Hyperinflammation in chronic granulomatous disease and anti-inflammatory role of the phagocyte NADPH oxidase. Semin Immunopathol 2008; 30: 255-271.

11 Magnani A, Brosselin P, Beauté J, et al. Inflammatory manifestations in a single-center cohort of patients with chronic granulomatous disease. J Allergy Clin Immunol 2014; 134: 655-662.

12 Mahdaviani SA, Mohajerani SA, Rezaei N, et al. Pulmonary manifestations of chronic granulomatous disease. Expert Rev Clin Immunol 2013; 9: 153-160.

13 Van den Berg JM, van Koppen E, Ahlin A, et al. Chronic granulomatous disease: the European experience. PLoS One 2009; 4: e5234.

14 Jones LB, McGrogan P, Flood TJ, et al. Chronic granulomatous disease in the United Kingdom and Ireland: a comprehensive national patient-based registry. Clin Exp Immunol 2008; 152: 211-218.

15 Segal BH, Romani LR. Invasive aspergillosis in chronic granulomatous disease. Med Mycol 2009; 47: Suppl. 1, S282-S290.

16 Moskaluk CA, Pogrebniak HW, Pass HI, et al. Surgical pathology of the lung in chronic granulomatous disease. Am J Clin Pathol 1994; 102: 684-691.

17 Gallin JI, Alling DW, Malech HL, et al. Itraconazole to prevent fungal infections in chronic granulomatous disease. N Engl J Med 2003; 348: 2416-2422.

18 Aguilar C, Malphettes M, Donadieu J, et al. Prevention of infections during primary immunodeficiency. Clin Infect Dis 2014; 59: 1462-1470.

19 Berendes H, Bridges RA, Good RA. A fatal granulomatosus of childhood: the clinical study of a new syndrome. Minn Med 1957; 40: 309-312.

20 Liese J, Kloos S, Jendrossek V, et al. Long-term follow-up and outcome of 39 patients with chronic granulomatous disease. J Pediatr 2000; 137: 687-693.

21 CEREDIH: The French PID study group. The French national registry of primary immunodeficiency diseases. Clin Immunol 2010; 135: 264-272.

22 Gathmann B, Grimbacher B, Beauté J, et al. The European internet-based patient and research database for primary immunodeficiencies: results 2006-2008. Clin Exp Immunol 2009; 157: Suppl. 1, 3-11.

23 De Pauw B, Walsh TJ, Donnelly JP, et al. Revised definitions of invasive fungal disease from the European Organization for Research and Treatment of Cancer/Invasive Fungal Infections Cooperative Group and the National Institute of Allergy and Infectious Diseases Mycoses Study Group (EORTC/MSG) Consensus Group. Clin Infect Dis 2008; 46: 1813-1821. 
24 Soncini E, Slatter MA, Jones LB, et al. Unrelated donor and HLA-identical sibling haematopoietic stem cel transplantation cure chronic granulomatous disease with good long-term outcome and growth. Br J Haematol 2009; 145: 73-83.

25 Martinez CA, Shah S, Shearer WT, et al. Excellent survival after sibling or unrelated donor stem cell transplantation for chronic granulomatous disease. J Allergy Clin Immunol 2012; 129: 176-183.

26 Khaldi H, Marchand-Adam S, Kannengiesser C, et al. Diffuse interstitial pneumonia and pulmonary hypertension: a novel manifestation of chronic granulomatous disease. Eur Respir J 2009; 33: 1498-1502.

27 Liese JG, Jendrossek V, Jansson A, et al. Chronic granulomatous disease in adults. Lancet 1996; 347: $220-223$.

28 Kuhns DB, Alvord WG, Heller T, et al. Residual NADPH oxidase and survival in chronic granulomatous disease. N Engl J Med 2010; 363: 2600-2610.

29 Blumental S, Mouy R, Mahlaoui N, et al. Invasive mold infections in chronic granulomatous disease: a 25 -year retrospective survey. Clin Infect Dis 2011; 53: e159-e169.

30 Segal BH, DeCarlo ES, Kwon-Chung KJ, et al. Aspergillus nidulans infection in chronic granulomatous disease. Medicine 1998; 77: 345-354.

31 Dotis J, Pana ZD, Roilides E. Non-Aspergillus fungal infections in chronic granulomatous disease. Mycoses 2013; 56: 449-462.

32 Henriet SS, Verweij PE, Warris A, et al. Aspergillus nidulans and chronic granulomatous disease: a unique hostpathogen interaction. J Infect Dis 2012; 206: 1128-1137.

33 Morgenstern DE, Gifford MA, Li LL, et al. Absence of respiratory burst in X-linked chronic granulomatous disease mice leads to abnormalities in both host defense and inflammatory response to Aspergillus fumigatus. J Exp Med 1997; 185: 207-218.

34 Brown KL, Bylund J, MacDonald KL, et al. ROS-deficient monocytes have aberrant gene expression that correlates with inflammatory disorders of chronic granulomatous disease. Clin Immunol 2008; 129: 90-102.

35 Romani L, Fallarino F, De Luca A, et al. Defective tryptophan catabolism underlies inflammation in mouse chronic granulomatous disease. Nature 2008; 451: 211-215.

36 Noel N, Mahlaoui N, Blanche S, et al. Efficacy and safety of thalidomide in patients with inflammatory manifestations of chronic granulomatous disease: a retrospective case series. J Allergy Clin Immunol 2013; 132: 997-1000.

37 Promteangtrong C, Salavati A, Cheng G, et al. The role of positron emission tomography-computed tomography/ magnetic resonance imaging in the management of sarcoidosis patients. Hell J Nucl Med 2014; 17: $123-135$.

38 Godoy MC, Vos PM, Cooperberg PL, et al. Chest radiographic and CT manifestations of chronic granulomatous disease in adults. AJR Am J Roentgenol 2008; 191: 1570-1575.

39 Cole T, Pearce MS, Cant AJ, et al. Clinical outcome in children with chronic granulomatous disease managed conservatively or with hematopoietic stem cell transplantation. J Allergy Clin Immunol 2013; 132: 1150-1155.

40 Güngör T, Teira P, Slatter M, et al. Reduced-intensity conditioning and HLA-matched haemopoietic stem-cell transplantation in patients with chronic granulomatous disease: a prospective multicentre study. Lancet 2014; 383 : $436-448$ 